Horizontes. Revista de Investigación en Ciencias de la Educación

ISSN 2616-7964

octubre-diciembre, 2017

Volumen 1, Número 4

pp. 28-36

www.revistahorizontes.org

\title{
Las innovaciones tecnológicas una herramienta de actualización en los consejos comunales. Una mirada de los actores sociales a la luz de la hermenéutica
}

\author{
Technological innovations an update tool in the communal councils. \\ A look by social actors in the light of hermeneutics \\ Jesús Alfredo Castillo Rondón \\ jesuscastillorondon@gmail.com \\ Universidad Nacional Experimental Simón Rodríguez
}

Recibido: 11-04-2017 / Revisado: 15-04-2017 / Aceptado: 17-05-2017 / Publicado: 09-10-2017

RESUMEN

La investigación realizada tuvo basada en interpretar las innovaciones tecnológicas utilizadas por los consejos comunales del municipio Pedro Zaraza; por lo que se enmarca en el paradigma interpretativo a través del método hermenéutico. La recolección de la información se realizó a través de un guion de preguntas aplicadas a los informante claves de la investigación utilizando como técnica para interpretar la información recopilada la categorización, contrastación y la triangulación que permitió interpretar lo recopilada y así construir una concepción sobre las categorías de estudio basada en la realidad.

Palabras clave: Innovaciones tecnológicas, Herramienta de actualización, Consejos comunales, Interpretación, Participación
ABSTRACT

The research has been based on the interpretation of the technological innovations used by the communal councils of the municipality Pedro Zaraza; Therefore, it is framed in the interpretative paradigm through the hermeneutic method. The collection of the information is done through a script of the questions applied to the key informants of the investigation using the technique for the interpretation the information collected the categorization, the contrasting and the selection that allowed the interpretation to compile and thus to construct a Conception on the category of study based on reality.

Key words: Technological innovations, Updating tool, Communal councils, Interpretation, Participation 
CONTEXTUALIZACIÓN DE LA REALIDAD

De la mano de los avances tecnológicos en las comunicaciones y el transporte, la globalización de la economía y los procesos de integración económica aparejados, conllevan cambios en la estandarización de los productos y en los gustos de los consumidores y redefinen, de esta forma, el marco en el cual las empresas planifican e implementan sus producciones. Y aún más, ante una economía globalizada, donde cada vez existe una mayor competencia internacional, es imprescindible esta redefinición para poder competir a nivel internacional. Así, las empresas afrontan la necesidad de redefinir sus ámbitos de actuación y adaptarse al incremento de la competencia en los mercados en los que ofrecen sus productos.

Tomando en cuenta lo expuesto por león (2011) quien señala: " cuando hablamos de tecnología solo vienen a la mente palabras como computadoras, juegos de videos, discos compactos, sin fijarnos que esta palabra se refiere al sin número de máquinas 0 herramientas movidas por una fuerza no humana pero que tiene que ser dirigida por el hombre; estas máquinas podrían ser, desde artículos tan pequeños como calculadoras o relojes, hasta automóviles, aviones, o las ya mencionadas y famosas computadoras sin embrago hay que señalar que varias de estas maquinarias trabajan por su cuenta pero fue un hombre quien las puso en marcha y quien la podrá detener". El mundo actual, se ha achicado debido a los avances de la tecnología moderna, tan es así, que ya se ha convertido en parte fundamental para el desarrollo funcional del país; hemos llegado al punto de que una sociedad sin tecnología es como una sociedad prehistórica.

Las innovaciones tecnológicas representan hoy en día una herramienta básica para el logro de una gerencia eficiente en organizaciones productivas. Su importancia radica en todos y cada uno de los procesos que una organización sólo puede llevar a cabo con eficiencia si cuenta con una apropiada base tecnológica, personal calificado en ciencia, tecnología, investigación y desarrollo, técnicas y métodos eficaces de adquisición, transferencia, comercialización y asimilación de tecnologías; todo lo cual es sumamente necesario para aumentar la productividad del negocio y por ende su rentabilidad.

En este sentido, las pequeñas, medianas y grandes empresas en los países del mundo industrializado han demostrado poseer un gran potencial y muestran evidencias reales, tanto de la generación de procesos innovativos, como de la creación, comercialización y transferencia continua de tecnología. Por su parte, según estudios realizados por Paredes (1996), los países en vías de desarrollo, Venezuela específicamente, presenta una creciente manifestación de iniciativas para la innovación tecnológica en pequeñas y medianas empresas, aunque ello no evidencie necesariamente la existencia de adecuados instrumentos de política industrial que favorezcan la viabilidad de la actividad innovativa.

Por consiguiente, los consejos comunales no pueden escapar de esta realidad ya que todos los procesos que realizan como organización son de forma manual y esto acarrea retraso en la información, generación de errores, alteración en la integridad de la base de datos, tiempos de espera elevados, incumplimiento de los objetivos planteados e insatisfacción a los miembros de la comunidad sobre las solicitudes realizadas.

\section{Interrogantes del estudio}

Bajo este argumento, se presenta esta investigación con el objetivo de interpretar las innovaciones tecnológicas como herramienta de actualización en los consejos comunales del municipio Pedro Zaraza del Estado Guárico. En tal sentido se plantea las siguientes interrogantes: 
¿Cuál es el grado de conocimiento que tienen los actores sociales sobre los consejos comunales?

¿Cuál es el modelo de gestión implementado por de los consejos del municipio Zaraza del Estado Guárico?

¿Qué importancia tiene la participación de los actores sociales de los consejos comunales del municipio Pedro Zaraza del Estado Guárico?

\section{Propósitos de la investigación}

- Develar el conocimiento que tienen los actores sociales sobre los consejos comunales.

- Interpretar los factores del entorno que inciden en las innovaciones tecnológicas del consejo comunal Chingoreto del municipio Zaraza del Estado Guárico

- Comprender la importancia de la participación de los actores sociales de los consejos comunales del municipio Pedro Zaraza del Estado Guárico

\section{Relevancia de la investigación}

En los últimos años se han desarrollado diversas iniciativas que tienden a la búsqueda de nuevas formas de explotar las considerables capacidades productivas creadas; las mismas apuntan hacia la reconversión tecnológica y organizativa de las empresas. Debido a que la reanimación de la economía conduce a una reestructuración de la producción y los servicios, donde se cuenten con estrategias bien creadas que permita la búsqueda de una mayor competitividad.

Actualmente resulta vital adaptar los procesos a través de las innovaciones tecnológicas para satisfacer las necesidades de los ciudadanos, lo cual, representa un desafío para los consejos comunales, especialmente las que se encuentran ubicadas en el municipio Pedro Zaraza del Estado Guárico.

\section{Estudios previos}

Sánchez M. (2012) en su trabajo de investigación titulado: El Fenómeno Social En Los Procesos Participativos De Los Consejos Comunales para optar al grado de Magister. Cuyo objetivo es interpretar el fenómeno social en los procesos participativos de los Consejos Comunales del Estado Carabobo, teniendo como centro que la participación en el seno de estas organizaciones es una condición indispensable para concebir al ciudadano como sujeto activo en la gestión de los asuntos públicos. Se inserta en una tipología documental, desde el enfoque cualitativo, utilizando para tal fin el método fenomenológico.

Para la realización del estudio se seleccionaron, tres (03) informantes claves de los consejos comunales de los municipios Naguanagua, Libertador y Valencia del Estado Carabobo, y estas organizaciones fueron seleccionadas según los siguientes criterios: Ser organizaciones sociales, por los años de constituidos. Estar inscritos en el Registro de Información Fiscal (R.I.F), que realicen reuniones periódicas, haber realizado un proyecto social o este en ejecución del mismo, poseer Código del Sistema Integral de Taquillas Únicas de Registro (SITUR) Consulta, por cuanto deben estar registrados ante el Ministerio del Poder Popular para las Comunas y Movimientos Sociales y deben poseer el Código del Sistema Integral de Taquillas Únicas de Registro (SITUR), Finalmente se llega a una reflexión, que el éxito de la participación ciudadana en estas organizaciones depende de la formación, grado de compromiso, trabajo en equipo, sentido de pertenencia, responsabilidad y la comunicación desde su cosmovisión cultural.

González (2012) realizo una tesis de maestría en la UNERG, titulada: Programa de orientación personal social dirigido a los voceros de los consejos comunales del municipio Pedro Zaraza para el logro de la participación protagónica en el desarrollo de 
su comunidad, cuyo objetivo fue proponer un programa de orientación personal social dirigido a los voceros de los consejos comunales del municipio Pedro Zaraza para el logro de la participación protagónica en el desarrollo de su comunidad. Las personas son esenciales para la organización y, ahora más que nunca, su importancia estratégica está en aumento, ya que todas las organizaciones compiten a través de su personal. El éxito de una organización depende cada vez más del conocimiento, habilidades y destrezas de sus trabajadores. Cuando el talento del recurso humano es valioso, raro y difícil de imitar y sobre todo organizado, una organización puede alcanzar ventajas competitivas que se apoyan en las personas. Enmarcado en una investigación de campo descriptivo, bajo la modalidad de proyecto factible.

La autora concluyo que los beneficios que tienen las organizaciones entre ellas las comunitarias, tal es el caso de los Consejos Comunales con la capacitación y adiestramiento se pueden enumerar los siguientes: Crear mejor imagen de los mismos, mejora la relación con los habitantes de la comunidad que representan, eleva la moral de la fuerza de trabajo, se incrementa la productividad y la calidad en el trabajo.

\section{Teorías de entrada}

Teoría de la Toma de Decisiones (KAST 1979)

Esta corriente de pensamiento, determina la administración desde una postura cuantitativa y supone los modelos matemáticos como herramientas básicas y fundamentales para la toma de decisiones, pues perspectivas cuantitativas repercuten en la productividad y la eficiencia de la organización. De igual forma, consideraba el uso de las computadoras como herramienta para la toma de decisiones, sobre todo por la interrelación entre la parte técnica con el factor humano; así mismo, hablaba de la racionalidad limitada, donde las decisiones eran tomadas con base en la experiencia, en los conocimientos y en la postura de la realidad, pero sin la comprensión total de la situación (Aktout, 1988, p. 252).

\section{Teoría General de Sistemas (Bertalanffy 1937)}

Teoría que contrarresta las evidencias de la brecha entre las disciplinas a fines, por lo tanto, promueve la tesis de las estructuras, en tanto que las estrategias promovidas por los sistemas u organizaciones tienden a cambiar, con base a los propios intereses. Las transformaciones de estructura y la teoría general de sistemas muestran la necesidad de crear conceptos, modelos y leyes aplicables a sistemas, sin importar su naturaleza física, biología y sociológica (Vertiz, 2008, p.17).

De esta forma, se da una visión holística de los sistemas. La teoría general de sistemas ayudo a la composición del conocimiento de diferentes disciplinas y edificó los principios para comprender las organizaciones y la administración como un sistema que interactúa con el ambiente que lo rodea. (Kast y Rosenzweig. 1981, p.130).

\section{Referentes teóricos}

\section{Los Consejos Comunales}

En la Ley de los Consejos Comunales 2006 las organizaciones comunitarias, "son las organizaciones que existen o pueden existir en las comunidades y que agrupan a un conjunto de ciudadanos y ciudadanas en base a objetivos e intereses comunes". (Art. 4, oral. 8).

\section{La participación}

Para algunos autores como Habermas (1990), la participación en el espacio público corresponde a un nivel ubicado entre la esfera privada y el Estado, cuya función principal no es sólo percibir e identificar problemas que afectan al conjunto de la sociedad, sino tematizarlos de manera convincente $y$ persuasiva para que sean asumidos y procesados por el sistema político. 
Según Pearse y Stifel (1980), la participación es considerada "como los esfuerzos organizados para aumentar el control sobre los recursos e instituciones sociales determinada por aquellos grupos y movimientos hasta ahora excluidos de dicho control" (p.65). De acuerdo con los autores la participación tiene como norte la incorporación de los grupos sociales excluidos.

\section{Innovación}

El termino innovar etimológicamente proviene del latín innovare, que quiere decir, cambiar o alterar las cosas introduciendo novedades (Medina y Espindola). Acerca del concepto innovación como Druker (1985) señalaba que la innovación es la herramienta especifica de los empresarios innovadores; el medio por el cual explorar el cambio como una oportunidad para un negocio diferente (p, $56)$.

\section{El proceso de innovación tecnológica}

La innovación es el proceso de generación de nuevas tecnologías. Ello implica tanto innovaciones de procesos como de productos. Es decir, una innovación de proceso (como se produce) supone la introducción de un nuevo método de producción o la modificación y mejoramiento de un proceso de producción o de gestión ya existente (Friedman, 1994). En el mismo sentido, la introducción de un nuevo producto o de una característica particular en un producto con el que el mercado no está familiarizado aún, supone una innovación de producto (que se produce).

\section{Referentes legales}

\section{Constitución de la República Bolivariana de Venezuela (1999)}

Artículo 62. Todos los ciudadanos y ciudadanas tienen el derecho de participar libremente en los asuntos públicos, directamente $o$ por medio de sus representantes elegidos o elegidas. La participación del pueblo en la formación, ejecución y control de la gestión pública es el medio necesario para lograr el protagonismo que garantice su completo desarrollo, tanto individual como colectivo. Es obligación del Estado y deber de la sociedad facilitar la generación de las condiciones más favorables para su práctica.

Artículo 110. El estado protegerá el interés público de la ciencia, la tecnología, el conocimiento, la innovación y sus aplicaciones y los servicios de información necesarios por ser instrumentos fundamentales para el desarrollo económico, social y político del país, así como para la seguridad y soberanía nacional. Para el fomento y desarrollo de esas actividades, el estado destinará recursos suficientes y creará el Sistema Nacional de Ciencia y Tecnología de acuerdo son la ley. El sector privado deberá aportar recursos para los mismos. El estado garantizara el cumplimiento de los principios éticos y legales que deben regir las actividades de investigación científica, humanista y tecnológica. La ley determinara los modos y medios para dar cumplimiento a esta garantía.

\section{Ley Orgánica de las Tecnologías de Información (2005)}

Artículo 17. Los órganos y entes del Poder Público deberán utilizar las nuevas tecnologías de información, tales como los medios electrónicos, informáticos y telemáticos, para su organización, funcionamiento, para su relación con los particulares y con los otros órganos y entes del Estado como medio para mejorar y transformar la gestión pública. 
Ley Orgánica de Ciencia, Tecnología e Información (2010)

Artículo 36. El ejecutivo nacional, a través de la autoridad nacional con competencia en materia de ciencia, tecnología, innovación y sus aplicaciones, diseñara e instrumentara los incentivos necesarios para estimular la formación e inserción de los cultores y cultoras científicos y tecnológicos en las unidades de producción social, los órganos adscritos a la autoridad nacional con competencia en materia de ciencia, tecnología de innovación y sus aplicaciones, así como en las instituciones universitarias que respondan a los proyectos que permitan resolver las necesidades concretas vinculadas al plan de desarrollo económico y social de la nación.

\section{Paradigma de la investigación}

La presente investigación se fundamenta en un paradigma interpretativo con un enfoque hermenéutico y un discurso cualitativo, porque las interrogantes se orientan hacia el conocimiento de las percepciones y vivencias de los sujetos en torno al comportamiento en un contexto específico.

\section{Método de la investigación}

Enmarcada en una investigación de carácter cualitativo, se aborda el método hermenéutico, donde Martínez (2010): "La hermenéutica aparece de manera explícita, pero en forma implícita está presente a lo largo de toda la investigación: en la elección del enfoque y de la metodología, en el tipo de preguntas que se formulan para recoger los datos, en la recolección de los datos y, en el análisis de dichos datos; todos estos pasos implican actividad interpretativa". (p.101)

De lo antes expuesto por el autor la misión de la hermenéutica es descubrir los significados de las cosas, interpretar lo mejor posible las palabras, los escritos, los textos y los gestos. Por ende, mediante la hermenéutica se pueden analizar una serie de análisis del fenómeno en estudio. Pero sin perder de vista su esencia enmarcada en todo aquello que lo rodea, fundamentado en los actores que hacen vida en el escenario a estudiar.

\section{Escenario de la investigación}

El escenario comprendió el lugar físico donde realicé la investigación. Según Shakespeare (2009) "el mismo hace referencia a la ecología de la investigación; Esto significa, al ambiente o medio socio geográfico donde se va a realizar ésta y de donde van a salir los informantes o población y muestra con los cuales se va a trabajar, a fin de obtener los datos necesarios para la investigación". En este caso, se delimita un espacio geográfico cultural que será el ámbito donde se moverá el investigador para llevarla a efecto. En este caso la investigación se desarrolla en el sector Chingoreto del Municipio Pedro Zaraza del Estado Guárico, donde funciona el consejo comunal, el mismo recibe a muchos deportistas ya que el sector cuenta con un estadio deportivo, algunas de sus calles son de arena, en el sector hace vida la Unidad Educativa Padre Francisco Hurtado que por muchos años viene formados a bachilleres. Las personas que forman parte del sector son respetuosas y trabajadoras, pero con un bajo nivel de educación.

\section{Informantes claves}

Para seleccionar a los informantes de esta investigación considere que tiene que ser una persona con amplio conocimiento sobre la trayectoria de los consejos comunales y la comunidad, identificado con sus raíces, costumbres, saberes, tecnologías, tradiciones y valores, conocedor de sus debilidades, fortalezas, amenazas y oportunidades, como también sobre las problemáticas prioritarias en donde viven. Según Taylor (1989), "el 
informante es una persona capaz de aportar información sobre el elemento de estudio y el portero, además de ser un informante cale, es una persona que se sitúa en el campo y ayuda en el proceso de selección de participantes en el caso de realizar entrevistas o grupos focales" (p 50). Los informantes del estudio son los miembros del consejo comunal o miembros de la comunidad del sector Chingoreto, capaces de aportar información relevante con el propósito de la investigación.

\section{La episteme}

Debido a que la investigación es de tipo cualitativa, siguiendo la metodología hermenéutica las técnicas a utilizar para la recolección de datos son la Observación Participante y la entrevista a profundidad. Para la observación participante utilizaré videograbadoras y notas de campos. Por otro lado, en relación al instrumento a utilizar en la entrevista a profundidad es un Guion de preguntas, el cual contemplará una serie de preguntas abiertas con el fin de que los sujetos significantes tengan la posibilidad de responder de forma amplia y en profundidad permitiendo recoger datos significativos para la investigación.

\section{Técnicas de Interpretación de la Información}

Para las técnicas de interpretación de la información y análisis de datos se implementarán las siguientes: categorización, contrastación y la triangulación.

\section{Se develan los hallazgos}

El apartado actual presenta la información recogida en las entrevistas realizadas a los informantes claves, con objeto de recabar información de los dado y aportado por los fenómenos del estudio ya descritos anteriormente como informantes claves con el fin de interpretar la información suministrada. Es oportuno destacar que, para ser buen uso de esta herramienta, a los textos crudos o entrevistas obtenidas se le aplicara el método hermenéutico, específicamente análisis del discurso, asumido en este estudio, debido a su pertenencia metodológica ya que permite dejar al descubierto las causas que originan el fenómeno, conocerlo, interpretarlo y comprenderlo, para luego realizar la categorización, contrastación y triangulación.

En la primera matriz se dispuso el corpus de la entrevista, la categoría predefinida continuando con los códigos, dando origen a un significado como aspectos más resultantes que permiten ir comprendiendo e interpretando más ampliamente las ideas de los sujetos de investigación.

Consecutivamente se efectuó el proceso de contratación, donde no sólo se busca entender mejor las posibles diferencias en los aportes de cada entrevistado, sino unificar los criterios que desprenden de los mismos lo cual hará posible una integración de la información recopilada, organizando y conceptualizando cada una de las categorías y sub categorías que recoge la investigación. Para finalizar se elaboró la triangulación, a través de una matriz integradora, entre los datos recopilados de los informantes, estudios paralelos o similares que se presentan en el estado del arte teórico y los aportes del investigador, lo cual permitió el surgimiento de una nueva percepción sobre la concepción y significado de las categorías objeto de estudio.

Una vez interpretado la información obtenida convertidos en hallazgos los cuales se encuentran enmarcados en función de los propósitos de la investigación los cuales guiaron el desarrollo del trabajo es importantes mencionar dichos propósitos los cuales marcaron el camino a recorrer: Develar el modelo de gestión implementado por el consejo comunal Chingoreto del municipio Zaraza del Estado Guárico; Interpretar los factores del entorno que inciden en las innovaciones tecnológicas del consejo comunal Chingoreto del municipio Zaraza del 
Estado Guárico y Comprender la importancia de la participación de los consejos comunales del municipio Pedro Zaraza del Estado Guárico.

Luego de aplicar el método hermenéutico para la producción del conocimiento emergente resultaron tres categorías lo cual permitió comprender la investigación las cuales son: los consejos comunales, innovaciones tecnológicas y la participación. Desde su fundamento teórico se llega a resultados obtenidos e interpretados lo que generan la construcción de las siguientes reflexiones:

En este sentido de las categorías que emergieron destaca los consejos comunales, Son aquellas organizaciones populares las cuales deciden reunirse para ver las necesidades más relevantes, donde el mismo pueblo es quien formula, ejecuta, controla y evalúa las políticas públicas, asumiendo así, el ejercicio real del poder popular en instancias de participación, articulación e integración entre los ciudadanos, ciudadanas y las diversas organizaciones comunitarias, movimientos sociales y populares, es decir, poniendo en práctica las decisiones adoptadas por la comunidad, por ello es preciso disponer de herramientas para la solución de conflictos en las circunstancias en que surjan para fortalecer la cultura política democrática y la calidad de las decisiones públicas.

Así mismo surge la siguiente categoría innovaciones tecnológicas, Son de vital importancia para la gestión administrativa, ya que todas las actividades que se realizan en el consejo comunal deben de llevar un esfuerzo ya que dentro de la organización ocurren una actividad que van aunados a un esfuerzo mancomunados de varias personas que integran la comunidad para la construcción del nuevo modelo de sociedad socialista de igualdad, equidad y justicia social. Son herramientas necesarias las cuáles permiten alcanzar las metas propuestas en la organización empresarial para lograr el desarrollo y fortalecimiento de la gestión, con el fin de manejar de manera transparente los recursos asignados al consejo comunal, y poder hacer una distribución de una manera correcta, lo que llevaría a que el consejo comunal tenga un buen desempeño en la gestión administrativa.

Y se finaliza con la categoría La participación, expresada a través las intervenciones en los procesos que interviene en los asuntos públicos que le afectan, permite canalizar el rumbo de la comunidad, de esta manera se puede ejercer el control, la vigilancia, la supervisión y la contraloría social a través de la gestión de los consejos comunales, es importante porque es el ejercicio donde el pueblo participa y forma parte de los asuntos públicos., al ejercer esta forma de participación se debe estar consciente de los obstáculos y resistencias a las que la comunidad se enfrenta, se busca desarrollar la eficacia y la eficiencia de los proyectos y aspiraciones de una comunidad.

\section{REFERENCIAS}

Arias, F. (2004). El proyecto de investigación 4o Edición. Caracas; Venezuela: Editorial Espíteme

Arias F. G. (2006). El proyecto de investigación. Introducción a la metodología científica. (5ta. Ed.). Caracas, Venezuela: Editorial Episteme

Constitución de la República Bolivariana de Venezuela. (2009). Gaceta Oficial de la República Bolivariana de Venezuela $\mathrm{N}^{\circ}$ 5.903. Febrero 19, 2009.

Chiavenato Idalberto. (2004). Iniciación a la Organización y el Control. Bogotá, Colombia: Editorial Nomos.

Etkin, Jorge Ricardo (2003) Gestión de la Complejidad de la Organizaciones. Editorial Oxford.

Guasch O. (1997). Observación Participante. Cuadernos metodológicos № 20, Madrid, España: CIS.

Hurtado, I, Toro, J. (2001). Paradigmas y Métodos de Investigación en Tiempos de Cambio. 4ta edición: Espíteme. Consultores y Asociados. 
Husserl, E. (1998). Invitación a la Fenomenología., Barcelona, España: Paidós

Hernández, R. Fernández, C. y Batista, P. (2006). Metodología de la Investigación. México, México: McGraw Hill.

Heidegger, M. (2000). Los problemas fundamentales de la fenomenología Estructuras y procesos. Serie Filosofía. Madrid: Trotta.

Heidegger, M. (2009). Ser y tiempo. Estructuras y procesos. Serie Filosofía (2a. ed.). Madrid, España: Trotta.

Husserl, E. (1962). Ideas relativas a una fenomenología pura y una filosofía fenomenológica. (2a. ed. en español.). México, México: Fondo de Cultura Económica.

Ley Orgánica de los Concejos Comunales (2009).

Ley del Plan de la Patria 2013- 2019. (2013). Publicada en Gaceta Oficial de la República Bolivariana de Venezuela № 6.118 del miércoles 4 de diciembre de 2013. Caracas.

Martínez, M. (2000). El paradigma emergente. Hacia una nueva teoría de la racionalidad científica. México, México: Trillas.

Méndez, C, (2006). Tecnologías y Herramientas de Gestión. Caso: grandes, medianas y pequeñas empresas en Bogotá. Bogotá, Colombia: Universidad del Rosario.

Méndez, C. (2006). Metodología. Diseño y desarrollo del proceso de investigación con énfasis en ciencias empresariales. Cuarta edición. Bogotá, Colombia: Limusa.

Méndez, C. (2006). Transformación cultural en las Organizaciones. Un Modelo para la
Gestión del Cambio. Bogotá, Colombia: Limusa.

Ricoeur, P. (1995). Teoría de la Interpretación: discurso y excedente de sentido. México [etc.]: Siglo Veintiuno.

Ricoeur, P. (2006). Del texto a la acción: ensayos de hermenéutica II. México, D.F. [etc.]: Fondo de Cultura Económica.

Ricoeur, P. (2008). Hermenéutica y acción. De la hermenéutica del texto a la hermenéutica de la acción. (3a. ed.). Buenos Aires, Argentina: Edit. Prometeo libros.

Rodríguez, G.,J. Gil y E. García. (1996). Metodología de la Investigación Cualitativa. Málaga: Aljibe

Rodríguez G, y otros. (1996). Metodología de la Investigación Cualitativa, Madrid, España: Ediciones Aljibe, pp.16-23.

Sabino, C. El Proceso de Investigación, Caracas, Editorial Panapo de Venezuela, S. A., 2002, pág.108.

Taylor, S., J. y Bogdan, R. (1992) Introducción a los métodos cualitativos de investigación. Barcelona: Paidós, p. 2

Todres, L. \& Holloway, I. (2006). Investigación fenomenológica. 5a. ed., pp. 224-237. Madrid: Mc GrawHill, Interamericana.

Gadamer, H.-G. (1977). Verdad y método: fundamentos de una hermenéutica filosófica. Salamanca: Ediciones Sígueme.

Van Manen, M. (2003). Investigación educativa y experiencia Vivida. Ciencia Humana para una pedagogía de la Acción y de la Sensibilidad. Idea Books. Barcelona, España 